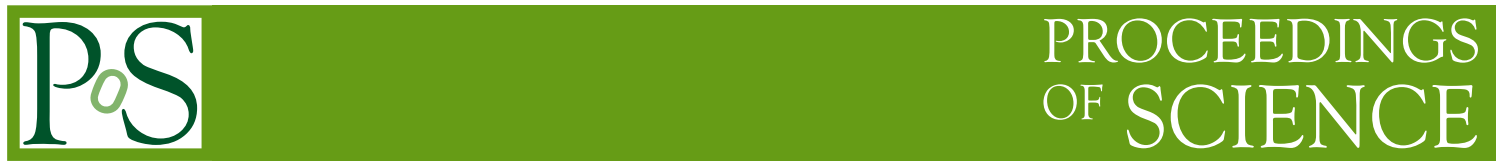

\title{
Thermodynamic quark susceptibilties in the PNJL model
}

\author{
M. Cristoforetti ${ }^{\star a b}$, T. Hell ${ }^{b}$, B. Klein ${ }^{b}$ and W. Weise ${ }^{b \dagger}$ \\ ${ }^{a} E C T^{*}$ I38100, Villazzano (TN), Italy \\ ${ }^{b}$ TU Munich, Physik-Department, D85747, Garching, Germany \\ E-mail: mcristofo@ect.it
}

\begin{abstract}
Applying Monte-Carlo methods to the Polyakov-loop extended Nambu-Jona-Lasinio (PNJL) model we go beyond the saddle-point approximation in a mean-field calculation and introduce fluctuations around the mean fields. The impact of fluctuations on the thermodynamics of the model in the two-flavor case becomes evident by studying the second-order Taylor expansion coefficients of the thermodynamic grand-canonical partition function with respect to the quark chemical potential. Here we show a comparison with extrapolations from lattice QCD. We find that in order to reproduce lattice data for the flavor non-diagonal quark susceptibilities the introduction of fluctuations is unavoidable.
\end{abstract}

The XXVIII International Symposium on Lattice Filed Theory

June 14-19,2010

Villasimius, Sardinia Italy

\footnotetext{
${ }^{*}$ Speaker.

${ }^{\dagger}$ Work supported in part by BMBF, GSI and by the DFG cluster of excellence Origin and Structure of the Universe.
} 


\section{Introduction}

Results of QCD thermodynamics from lattice computations are reproduced surprisingly well with a quasiparticle model, an extension of the Nambu-Jona-Lasinio model with inclusion of Polyakov-loop dynamics (the PNJL model) at the mean-field level [1, 2, 3]. A better understanding of the mechanism at the origin of these transitions requires the investigation of fluctuations in the PNJL model [4]. Here we include fluctuations by performing numerical simulations of the thermodynamics using standard Monte-Carlo (MC) techniques. The advantage of this method is that it automatically incorporates fluctuations to all orders. In the present work we restrict ourselves to fluctuations of the static zero-modes which lead to an improvement beyond the saddle-point approximation.

We perform our analysis for the case of vanishing chemical potential where a comparison with lattice simulation results is possible. In particular, we will see that the temperature dependence of the flavor non-diagonal second derivative of the thermodynamic grand-canonical partition function, with respect to quark chemical potentials, is very sensitive to these fluctuations.

\section{The PNJL partition function}

The Euclidean action of the two-flavor PNJL model including finite baryon and isospin chemical potentials is given by [5]

$$
\begin{aligned}
\mathscr{S}_{E}(\psi, \bar{\psi}, \phi)= & \int_{0}^{\beta} \mathrm{d} \tau \int \mathrm{d}^{3} x\left\{\bar{\psi}\left(i \mathbb{D}+\gamma_{0} \tilde{\mu}-\mathbf{m}\right) \psi+G\left[(\bar{\psi} \psi)^{2}+\left(\bar{\psi} i \gamma_{5} \vec{\tau} \psi\right)^{2}\right]\right\} \\
& -\beta \int \mathrm{d}^{3} x \mathscr{U}(\phi, \beta),
\end{aligned}
$$

with $\beta=1 / T$. Here $\psi$ is the $N_{f}=2$ doublet quark field, $\mathbf{m}=\operatorname{diag}\left(m_{u}, m_{d}\right)$ is the quark mass matrix and the covariant derivative is

$$
i \not D=i \gamma_{\mu}\left(\partial^{\mu}-i g A^{\mu}\right) .
$$

The quark chemical-potential matrix, $\tilde{\mu}$, is defined as $\tilde{\mu}=\operatorname{diag}\left[\mu_{u}, \mu_{d}\right]$.

The Polyakov-loop effective potential, $\mathscr{U}$, involves the gauge-field degrees of freedom denoted by $\phi$ and models the confinement-deconfinement transition in the pure gauge theory at mean-field level. In the PNJL model quarks interact with a background color gauge field $A_{4}=i A_{0}$, where $A_{0}=\delta_{\mu 0} g \mathscr{A}_{a}^{\mu} t^{a}$ with the gluon fields $\mathscr{A}_{a}^{\mu} \in \mathrm{SU}(3)_{c}$ and $t^{a}=\lambda^{a} / 2$. The field $A_{4}$ is related to the traced Polyakov loop according to

$$
\Phi=\frac{1}{N_{c}} \operatorname{tr}_{c} L \quad \text { with } \quad L=\exp \left(i \int_{0}^{\beta} \mathrm{d} \tau A_{4}\right) .
$$

In Polyakov gauge, the matrix $L$ is given in a diagonal representation

$$
L=\exp \left(i\left(\phi_{3} \lambda_{3}+\phi_{8} \lambda_{8}\right)\right)
$$

with the (diagonal) SU(3) generators $\lambda_{3}$ and $\lambda_{8}$. The dimensionless effective fields $\phi_{3}$ and $\phi_{8}$ are identified with the Euclidean gauge fields in temporal direction divided by the temperature, $A_{4}^{(3)} / T$ and $A_{4}^{(8)} / T$. These two fields parametrize the diagonal elements of $\mathrm{SU}(3)_{c}$. 
In this paper we consider the ansatz for the effective potential given in $[6,7]$ motivated by the $\mathrm{SU}(3)$ Haar measure which emerges when integrating out six of the eight gluon fields:

$$
\frac{\mathscr{U}\left(\Phi, \Phi^{*}, T\right)}{T^{4}}=-\frac{1}{2} a(T) \Phi^{*} \Phi+b(T) \ln \left[1-6 \Phi^{*} \Phi+4\left(\Phi^{* 3}+\Phi^{3}\right)-3\left(\Phi^{*} \Phi\right)^{2}\right] .
$$

The temperature-dependent prefactors are given by

$$
a(T)=a_{0}+a_{1}\left(\frac{T_{0}}{T}\right)+a_{2}\left(\frac{T_{0}}{T}\right)^{2} \quad \text { and } \quad b(T)=b_{3}\left(\frac{T_{0}}{T}\right)^{3} .
$$

The particular choice of $a(T)$ and $b(T)$ is such that we can reproduce the high-temperature behavior of thermodynamic quantities like pressure, energy and entropy density. An additional constraint for fixing the parameters is the critical temperature of the first-order deconfinement transition in pure gauge QCD, $T_{0}=270 \mathrm{MeV}$, as given by lattice calculations, and the requirement that $\Phi^{*}, \Phi \rightarrow 1$ as $T \rightarrow \infty$.

Given the action (2.1), the partition function of our system is

$$
\mathscr{Z}=\mathscr{N} \int \mathscr{D} \phi \mathscr{D} \sigma \mathscr{D} \vec{\pi} \exp \left[\operatorname{Tr} \ln \left[S^{-1}\right]-\frac{1}{T} \int \mathrm{d}^{3} x\left(\mathscr{U}(\phi, T)+\frac{\sigma^{2}+\vec{\pi}^{2}}{2 G}\right)\right] .
$$

where $\phi$ stands for the Polyakov loop fields $\phi_{3}$ and $\phi_{8}$. We write the pion field $\vec{\pi}=\left(\pi^{1}, \pi^{2}, \pi^{3}\right)$ in terms of $\pi^{ \pm}=\frac{1}{\sqrt{2}}\left(\pi^{1} \pm i \pi^{2}\right), \pi^{0}=\pi^{3}$ and $\tau^{ \pm}=\frac{1}{2}\left(\tau^{1} \pm i \tau^{2}\right)$, so that

$$
\vec{\tau} \cdot \vec{\pi}=\sqrt{2}\left(\tau^{+} \pi^{-}+\tau^{-} \pi^{+}\right)+\tau^{3} \pi^{0} .
$$

The inverse quark propagator takes the form

$$
S^{-1}=\left(\begin{array}{cc}
-\partial+\left(\mu_{u}-i A_{4}\right) \gamma_{0}+i \gamma_{5} \pi^{0}-M & i \sqrt{2} \gamma_{5} \pi^{+} \\
i \sqrt{2} \gamma_{5} \pi^{-} & -\partial+\left(\mu_{d}-i A_{4}\right) \gamma_{0}+i \gamma_{5} \pi^{0}-M
\end{array}\right)
$$

with the dynamical quark mass $M=m_{0}-\sigma$ generated by the scalar field $\sigma<0$. We work in the isospin-symmetric limit with $m_{0}=m_{u}=m_{d}$ for convenience. This scalar field is related to the chiral (quark) condensate by $\sigma=G\langle\bar{\psi} \psi\rangle$.

\section{PNJL model in a finite volume}

In the present calculation we perform a step beyond mean-field approximation by including fluctuations of the zero modes of the relevant fields. This is admittedly only part of all possible field fluctuations, but it represents nevertheless an improvement with respect to the standard meanfield calculation. These zero-mode fluctuations can be introduced considering a system defined in a finite volume $V$.

The partition function in momentum space is written as

$$
\mathscr{Z}=\int \mathscr{D} \phi \mathscr{D} \sigma \mathscr{D} \vec{\pi} \exp \left[\frac{V}{T}\left(\sum_{n} \sum_{\vec{p}} \operatorname{Tr} \ln \left[S^{-1}\left(i \omega_{n}, \vec{p}\right)\right]-\mathscr{U}(\phi, T)-\frac{\sigma^{2}+\vec{\pi}^{2}}{2 G}\right)\right]
$$

where $\omega_{n}=(2 n+1) \pi T$ are the Matsubara frequencies. 
The presence of a volume factor $V$ in the exponent of Eq. (3.1) makes it possible to compute the full partition function in mean-field approximation using Monte-Carlo techniques. The size of the volume is now specified according to the conventions adopted in lattice calculations. For a fixed extension of the lattice in the Euclidean time direction, the temperature is set by the lattice spacing $a$, and the volume size is related to the temperature:

$$
a=\frac{1}{N_{t} T} \quad \Longrightarrow \quad V=N_{s}^{3} a^{3}=\frac{N_{s}^{3}}{N_{t}^{3} T^{3}},
$$

where $N_{t}$ is the number of lattice sites in the Euclidean time direction, and $N_{s}$ is the number of lattice sites in the space direction. It follows that $V=k / T^{3}$, where different values of $k=\left(N_{s} / N_{t}\right)^{3}$ will be chosen for our purpose: $k=64,125,250,500,1000,2500$. The ratio between the smallest and the largest $k$ is approximately 40. In this way we can study systematically the dependence of the observables on the volume size at fixed temperature $T$. The typical lattice simulation volume corresponds to $k=64$.

\section{Non-zero quark chemical potentials: Taylor expansion}

The starting point for studying the thermodynamics for $N_{f}=2$ quark flavors is the partition function (3.1). The degrees of freedom in this case are the $A_{4}^{(3)}$ and $A_{4}^{(8)}$ components of the gauge field and the bosonic field variables $\sigma$ and $\vec{\pi}$. In the NJL sector of the model we need to specify the current quark mass $m_{0}$, the coupling constant $G$ and the three-momentum cutoff $\Lambda$. The parameters used here are the ones of Refs. [5, 6]:

$$
m_{0}=5.5 \mathrm{MeV}, G=10.1 \mathrm{GeV}^{-1}, \Lambda=650 \mathrm{MeV} .
$$

These parameters are determined to reproduce pion properties (mass, decay constant) in the infinite volume limit at zero temperature.

Dealing with non-zero quark chemical potentials $\mu_{q}$ in lattice QCD thermodynamics is notoriously difficult because of the well-known fermion sign problem. A possible way of overcoming this problem is the Taylor-expansion approach. Instead of performing an explicit calculation at $\mu_{q} \neq 0$, the thermodynamic potential is expanded in a Taylor series in $\mu_{q} / T$ around zero chemical potential,

$$
\Omega(T, \mu)=\frac{1}{V T^{3}} \ln \mathscr{Z}=\sum_{i, j=0}^{\infty} \chi_{i j}(T)\left(\frac{\mu_{u}}{T}\right)^{i}\left(\frac{\mu_{d}}{T}\right)^{j}
$$

with

$$
\chi_{i j}(T)=\left.\frac{1}{i ! j !} \frac{\partial^{i+j} \Omega}{\partial\left(\mu_{u} / T\right)^{i} \partial\left(\mu_{d} / T\right)^{j}}\right|_{\mu_{u}=\mu_{d}=0},
$$

where only even terms survive due to $C P$ symmetry. The coefficients $\chi_{i j}(T)$ are evaluated at $\mu_{q}=0$.

The comparison between lattice data and Monte-Carlo calculations for these coefficients in the PNJL model represent an important test of this model. In particular, the flavor non-diagonal coefficient $\chi_{11}$ that vanishes in the saddle-point approximation is of interest in this context: it is necessary to take fluctuations of the mean field into account in order to obtain a non-vanishing result for $\chi_{11}$. 


\subsection{Second order Taylor expansion coefficients and susceptibilities}

From the definition (4.2) we obtain the coefficients $\chi_{u u}$ and $\chi_{u d}$ (quark susceptibilities)

$$
\begin{aligned}
\chi_{u q}= & \frac{1}{V T} \frac{\partial^{2}}{\partial \mu_{u} \partial \mu_{q}} \ln \mathscr{Z}=\frac{T^{2}}{V T^{3}}\left(\frac{V}{T}\left\langle\frac{\partial^{2}}{\partial \mu_{u} \partial \mu_{q}} \ln \operatorname{det} S^{-1}\left(T, \mu_{u}, \mu_{d}, \sigma, \vec{\pi}, A\right)\right\rangle\right. \\
& +\left(\frac{V}{T}\right)^{2}\left\langle\left(\frac{\partial}{\partial \mu_{u}} \ln \operatorname{det} S^{-1}\left(T, \mu_{u}, \mu_{d}, \sigma, \vec{\pi}, A\right)\right)^{2}\right\rangle \\
& \left.-\left(\frac{V}{T}\right)^{2}\left\langle\frac{\partial}{\partial \mu_{u}} \ln \operatorname{det} S^{-1}\left(T, \mu_{u}, \mu_{d}, \sigma, \vec{\pi}, A\right)\right\rangle^{2}\right) .
\end{aligned}
$$

The behavior of the flavor non-diagonal coefficient $\chi_{11}=\chi_{u d}$ is quite different from that of all the other expansion coefficients: it vanishes in the saddle-point approximation whereas lattice QCD clearly displays a non-zero signal for this quantity around $T_{c}$.

From a detailed analysis of (4.3) one can find that there are two main contributions to signal in this channel: one is connected with fluctuations of $A_{8}$, the other with fluctuations of the pion field. Both of these give zero in the saddle-point limit.

The pionic and $A_{8}$ contributions to $\chi_{u d}$ resulting from the MC-PNJL computation are shown in Fig. 1. Two characteristic features are immediately apparent. First, the term involving pionic zeromodes is strongly volume dependent and vanishes in the limit of infinite volume. Secondly, the term associated with fluctuations of the $A_{8}$ gauge field is independent of the box size and survives in fact as the volume becomes infinitely large.
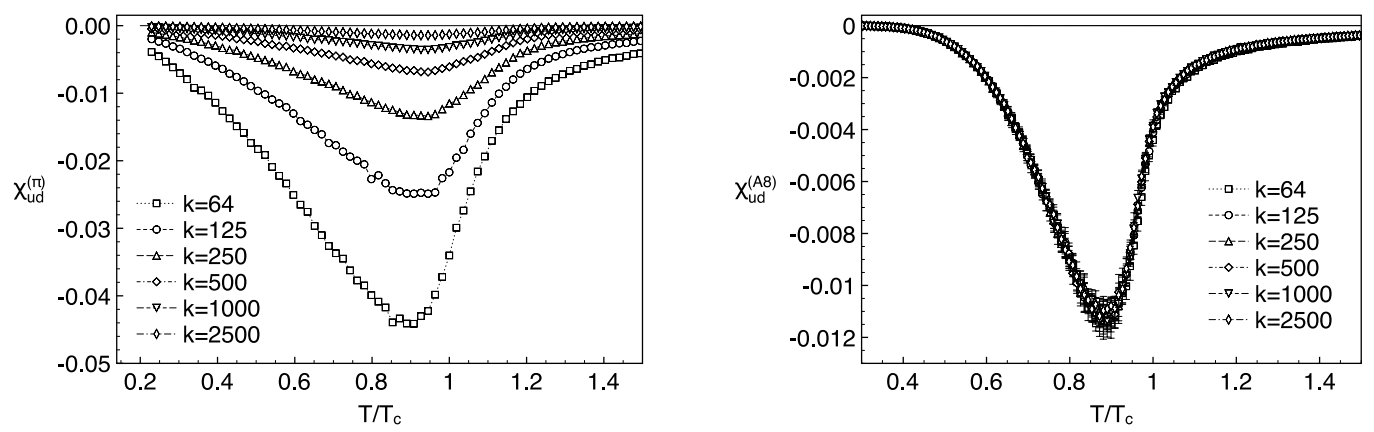

Figure 1: Different contributions to the off-diagonal susceptibility $\chi_{11}=\chi_{u d}$ for different volume ratios $k$ computed in the Monte-Carlo approach. Left panel: Contribution from pionic fluctuations, for which the volume dependence is large. Right panel: Contribution from fluctuations of the $A_{8}$ field, which show a negligible volume dependence.

\subsection{Chiral effective Lagrangian}

In order to better understand the role of the pionic fluctuations in the evaluation of $\chi_{u d}$, let us briefly digress and study this issue in the context of chiral perturbation theory (ChPT).

For low temperatures and small values of the chemical potential, the physics is dominated by the effects of light pions and we can describe the system in terms of an effective chiral Lagrangian 
which expanded to second order in the pion fields $\pi^{a}$ is given by

$$
\begin{aligned}
\mathscr{L}= & \frac{1}{2}\left(\partial_{v} \pi^{a}\right)\left(\partial_{v} \pi^{a}\right)+i 2 \mu_{I} f_{\pi}\left(\partial_{0} \pi^{3}\right)+i 2 \mu_{I}\left[\left(\partial_{0} \pi^{1}\right) \pi^{2}-\left(\partial_{0} \pi^{2}\right) \pi^{1}\right] \\
& +\frac{1}{2} m_{\pi}^{2} \pi^{a} \pi^{a}-2 \mu_{I}^{2}\left(\pi^{1} \pi^{1}+\pi^{2} \pi^{2}\right) .
\end{aligned}
$$

In order to make contact with the results from the Monte-Carlo evaluation of the PNJL model with fluctuations of the mean field only, it is sufficient to take the static part of the Lagrangian into account. The partition function for this static part is

$$
\mathscr{Z}_{\text {static }}=\int \prod_{a=1}^{3} d \pi^{a} \exp \left\{-\frac{V}{T}\left[\frac{1}{2} m_{\pi}^{2} \pi^{a} \pi^{a}-4 \mu_{I}^{2}\left(\pi^{+} \pi^{-}\right)\right]\right\} .
$$

From the definition of $\chi_{u d}(4.3)$ we have

$$
\chi_{u d}^{(\pi)}=-\frac{2}{V T m_{\pi}^{2}}=-\frac{2 T^{2}}{k} \frac{1}{m_{\pi}^{2}},
$$

setting again $V=k / T^{3}$. This prediction can be compared directly with our Monte-Carlo PNJL results, provided we take the temperature dependence of the pion mass into account, using the relation given in [8].

Fig. 2 (right panel) demonstrates that the picture so obtained from the chiral effective Lagrangian is completely consistent with our Monte-Carlo calculations in the PNJL model, as far as the pionic contributions to $\chi_{u d}$ are concerned. From the figure it also follows that the chiralperturbation-theory prediction for this coefficient is reliable until around $T / T_{c} \simeq 0.7$.
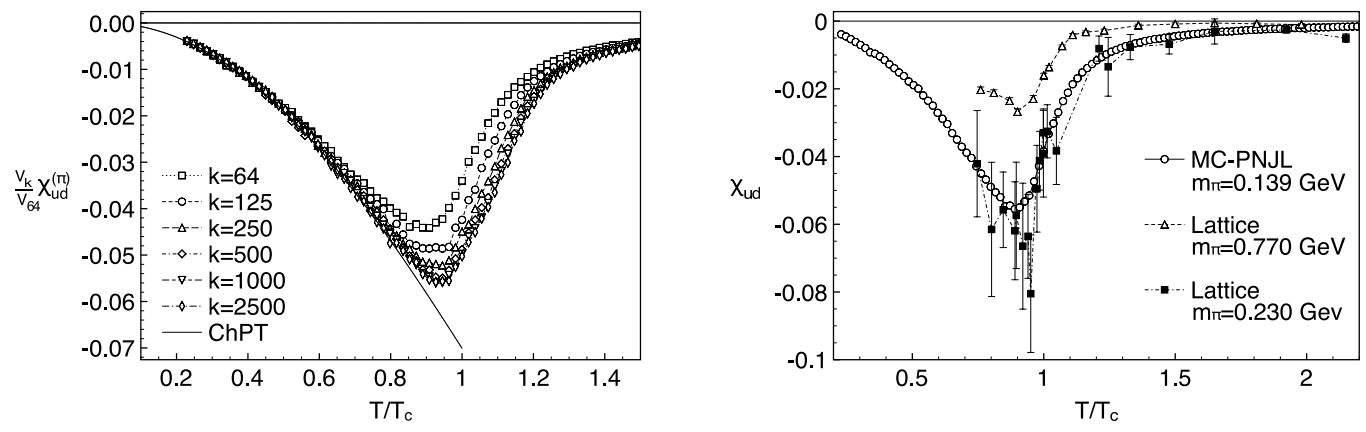

Figure 2: Right panel: scaled pionic contribution to the off-diagonal susceptibility compared with the ChPT prediction. All Monte-Carlo PNJL results are multiplied by the volume $V_{k} / V_{64}$ and therefore scale with the $k=64$ curve, using Eq. (4.6). Left panel: temperature dependence of the flavor off-diagonal susceptibility $\chi_{u d}$ in the Monte-Carlo approach to the PNJL model, using $k=64(L T=4)$. Lattice data $[9,10]$ with the same volume aspect ratio $L T$ and different pion masses are also shown for orientation.

\subsection{Comparison with lattice data}

Lattice-QCD studies of $\chi_{u d}$ have been carried out for example in Refs. [9, 10], both with $k=64$ but with different quark masses, corresponding to pion masses $m_{\pi}=230 \mathrm{MeV}$ and $770 \mathrm{MeV}$. These lattice results are compared to our Monte-Carlo PNJL computations (using the physical pion mass) 
in Fig. 2. The shape of the $\chi_{u d}$ signal is quite well reproduced within the large error band of the lattice data. The difference between lattice results computed with different pion masses is now quite plausible. Given that the pionic fluctuations dominate over those from the $A_{8}$ component of the Polyakov-loop field, this behavior is just what one expects from Eq. (4.6). At the same time, one would expect that lattice simulations performed ideally with physical quark masses would actually yield even larger magnitudes of $\chi_{u d}$ than those with $m_{\pi}=230 \mathrm{MeV}$. The Monte-Carlo results notably include only the pionic zero modes. Finite-momentum fluctuations would tend to further increase the pionic effects in $\chi_{u d}$.

\section{Conclusions}

In this work we have applied standard Monte-Carlo techniques to a PNJL model in order to go beyond the saddle-point approximation. This becomes important when the system is considered in a finite volume. The strength of the fluctuations introduced in this way depends on the size of the volume.

The inclusion of such beyond-mean- field fluctuations in a finite volume does affect the susceptibilities significantly. We find that their impact is crucial for the evaluation of higher-order Taylor expansion coefficients of the pressure. Our result from a Monte-Carlo computation agrees well with lattice data using the same $k$ for the Euclidean volume. The role of pionic zero-mode fluctuations is clarified showing fully consistent results with those from chiral perturbation theory for temperatures below $T_{c}$.

\section{References}

[1] K. Fukushima, Relation between the Polyakov loop and the chiral order parameter at strong coupling, Phys. Rev. D 68 (045004) [hep-ph/0303225].

[2] C. Ratti et al., Phases of QCD: Lattice thermodynamics and a field theoretical model, Phys. Rev. D 73 (014019) [hep-ph/0506234].

[3] T. Hell et al., Dynamics and thermodynamics of a non-local PNJL model with running coupling, Phys. Rev. D 79 (014022) [0810.1099].

[4] M. Cristoforetti et al., Thermodynamics and quark susceptibilities: a Monte-Carlo approach to the PNJL model, Phys. Rev. D 81 (114017) [arXiv:10 02.2336].

[5] S. Roessner et al., The chiral and deconfinement crossover transitions: PNJL model beyond mean field, Nucl. Phys. A 814 (118) [0 712 .3152].

[6] S. Roessner et al., Polyakov loop, diquarks and the two-flavour phase diagram, Phys. Rev. D 75 (034007) [hep-ph/0609281].

[7] C. Ratti et al., Thermodynamics of the PNJL model, Eur. Phys. J. C 49 (213) [hep-ph / 0609218 ].

[8] J. Gasser and H. Leutwyler, Light Quarks at Low Temperatures, Phys. Lett. B 184 (83).

[9] C. R. Allton et al., Thermodynamics of two flavor QCD to sixth order in quark chemical potential, Phys. Rev. D 71 (054508) [hep-lat/ 0501030 ].

[10] R. V. Gavai and S. Gupta, QCD at finite chemical potential with six time slices, Phys. Rev. D 78 (114503) [0806.2233]. 